\title{
Computational and Experimental Simulations of the Flow Characteristics of an Aerospike Nozzle
}

\author{
G. Rajesh*, Gyanesh Kumar**, H D Kim***† and Mathew George****
}

\begin{abstract}
Single Stage To Orbit (SSTO) missions which require its engines to be operated at varying back pressure conditions, use engines operate at high combustion chamber pressures (more than 100bar) with moderate area ratios (AR 70 80). This ensures that the exhaust jet flows full during most part of the operational regimes by optimal expansion at each altitude. Aero-spike nozzle is a kind of altitude adaptation nozzle where requirement of high combustion chamber pressures can be avoided as the flow is adapted to the outside conditions by the virtue of the nozzle configuration. However, the thrust prediction using the conventional thrust equations remains to be a challenge as the nozzle plume shapes vary with the back pressure conditions. In the present work, the performance evaluation of a new aero-spike nozzle is being carried out. Computational studies are carried out to predict the thrust generated by the aero-spike nozzle in varying back pressure conditions which requires the unsteady pressure boundary conditions in the computational domain. Schlieren pictures are taken to validate the computational results. It is found that the flow in the aero-spike nozzle is mainly affected by the base wall pressure variation. The aerospike nozzle exhibits maximum performance in the properly expanded flow regime due to the open wake formation.
\end{abstract}

Key Words: Aerospike, Closed wake, recirculation, open wake, thrust coefficient, base pressure

\section{Introduction}

Conventional bell nozzle with fixed area ratio works efficiently at a particular altitude where exit pressure exactly matches with the back (ambient) pressure causing exhaust to perfectly expanded thereby maximizing efficiency and thrust. At lower altitude where ambient pressure is higher than the exhaust pressure, the ambient air pushes the exhaust air inward which causes the separation of flow from the boundary of nozzle wall thereby decreasing thrust and efficiency. This condition is also known as overexpansion. At higher altitude where ambient pressure is lower than the exit pressure which is

Assistant Professor, Keimyung University

$\dagger$ Professor, Andong National University

E-mail : kimhd@andong.ac.kr

*Scientist, RAMD/SRG, ISAC, Bangalore, ISRO, India

**Scientist, RAMD/SRG, ISAC, Bangalore, ISRO, India

$* * * *$ Scientist, LPSC, Trivandrum, ISRO, India known as under expansion. Ambient air causes exhaust to flow past the nozzle exit. Since the additional expansion occurs outside of the nozzle, the available thrust in the nozzle is lost. Since, both overexpansion and under expansion reduce overall engine efficiency and thrust, for maximizing thrust and efficiency, the concept of an ideal nozzle has been contemplated $^{(1)}$.

An ideal nozzle would be able to continually adjust its area ratio to maximize thrust at each altitude, known as altitude compensation. Practically it is not possible to design a bell nozzle which changes its geometry during the flight. Aero-spike nozzle ${ }^{(2)}$ is an option where the flow is adapted to the ambient conditions at all altitudes, by virtue of the nozzle configuration. In theory at least, the aerospike nozzle meets or exceeds the performance of the bell nozzle at all operating pressures ${ }^{(3)}$.

Though the aero-spike nozzle has many advantages such as, uniform load distribution, simple gas dynamic configuration, and flexible structure combinations, the 
realization of such nozzle faces many hurdles due to its aerodynamic and structural design considerations ${ }^{(4)}$. To contain the extreme heating loads on the annular throat and nozzle, a combination of regenerative cooling, film cooling and dump cooling using hydrogen has to be employed. Also the thrust prediction remains a challenge as the nozzle plume shapes vary with the back pressure conditions where the conventional thrust equations fail to predict the thrust ${ }^{(5)}$. Performance validation is not yet done, although experiments are being done around the world ${ }^{(6)}$.

The present work is hence planned to predict the thrust developed in an annular thrust chamber Aerospike nozzle at varying back pressure conditions which requires the unsteady pressure boundary conditions to be imposed at the outlets. The performance of the nozzle is planned to be analyzed using a computational model which employ an unsteady simulation to account for the varying back pressure conditions to match with the actual flight data. Flow visualization is also planned to qualitatively asses the flow field and to validate the computational results.

\section{Computational methodology}

The flow field in ejector is governed by compressible axis symmetric Navier-Stokes equation. The equations below however are written in Cartesian co-ordinates for easiness. In this study Favre averaged NS equations are used because of their pertinence in density varying flows. The equations hence are written below. The turbulence is modeled by modeling the Reynolds stresses. The summary of equations used for this which is given below.

Continuity

$$
\frac{\partial \rho}{\partial t}+\frac{\partial}{\partial x_{i}}\left(\rho u_{i}\right)=0
$$

Momentum

$$
\begin{aligned}
\rho \frac{D\left(u_{i}\right)}{D t}=-\frac{\partial \rho}{\partial x_{i}}+ & \frac{\partial}{\partial x_{j}}\left[\mu_{\mathrm{eff}}\left(\frac{\partial u_{i}}{\partial x_{j}}+\frac{\partial u_{j}}{\partial x_{i}}-\frac{2}{3} \delta_{i j} \frac{\partial u_{k}}{\partial x_{k}}\right)\right] \\
& +\frac{\partial}{\partial x_{j}}\left(-\rho \overline{u_{i}^{\prime} u_{j}^{\prime}}\right)
\end{aligned}
$$

The velocity here is mean velocity, while the prime components are the disturbance components (due to turbulence) and it denotes Reynolds stresses.

$$
\begin{aligned}
\frac{\partial}{\partial t}(\rho E) & +\frac{\partial}{\partial x_{i}}\left[u_{i}(\rho E+P)\right] \\
= & \frac{\partial}{\partial x_{i}}\left[\left(\alpha+\frac{C_{p} \mu_{t}}{P_{r t}}\right) \frac{\partial T}{\partial x_{i}}+u_{j}\left(\tau_{i j}\right)_{\mathrm{eff}}\right]
\end{aligned}
$$

$\mathrm{E}$ and $\mathrm{T}$ are mass averaged, while $\tau_{\mathrm{ij}}$ is the stress tensor, where

$$
\tau_{i j}=\mu_{\mathrm{eff}}\left(\frac{\partial u_{j}}{\partial x_{i}}+\frac{\partial u_{i}}{\partial x_{j}}\right)-\frac{2}{3} \mu_{\mathrm{eff}} \frac{\partial u_{i}}{\partial x_{i}} \delta_{i j}
$$

The ideal gas equation is also used for balancing the equations with unknown

$$
\frac{P}{\rho}=r T
$$

The transport equation for Reynolds stress, which are computed for Reynolds stresses in RSM model is

$$
\begin{aligned}
& \frac{\partial}{\partial t}\left(\rho \overline{u_{i}^{\prime} u_{j}^{\prime}}\right)+\frac{\partial}{\partial x_{k}}\left(u_{k} \rho \overline{u_{i}^{\prime} u_{j}^{\prime}}\right) \\
& \quad=D_{i j}^{T}+D_{i j}^{L}+P_{i j}+\phi_{i j}+\varepsilon_{i j}
\end{aligned}
$$

The right hand side denotes turbulent diffusion, molecular diffusion, stress production, pressure strain, and dissipation respectively, where,

$$
\begin{aligned}
& D_{i j}^{L}=\frac{\partial}{\partial x_{k}}\left(\mu \frac{\partial}{\partial x_{k}} \overline{u_{i} u_{j}}\right) \\
& P_{i j}=-\rho\left(\overline{u_{i} u_{j}} \frac{\partial u_{j}}{\partial x_{k}}+\overline{u_{i} u_{j}} \frac{\partial u_{i}}{\partial x_{k}}\right)
\end{aligned}
$$

The equations for other parameters are not known and have to be modeled, the modeled equations are,

$$
D_{i j}^{T}=-\frac{\partial}{\partial x_{k}}\left[\rho \overline{u_{i} u_{j} u_{k}}+\overline{P\left(\delta_{k j} u_{i}+\delta_{i k} u_{j}\right)}\right]
$$

$$
\phi_{i j}=\overline{P\left(\frac{\partial u_{i}}{\partial x_{j}}+\frac{\partial u_{j}}{\partial x_{i}}\right)}
$$




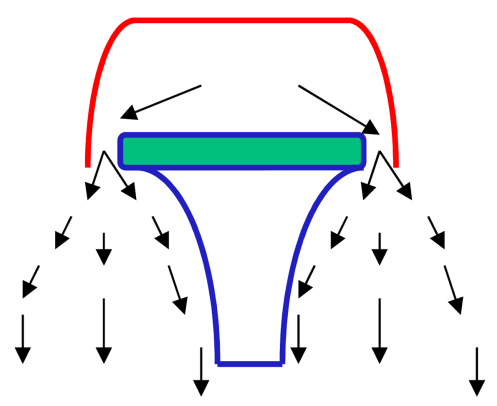

Fig. 1. Schematic of aerospike nozzle.

$$
\varepsilon_{i j}=-2 \mu \overline{\frac{\partial u_{i}}{\partial x_{k}} \frac{\partial u_{j}}{\partial x_{k}}}
$$

\subsection{Boundary conditions}

A schematic of the aerospike nozzle is shown in Fig. 1.

An axisymmetric model of the aerospike nozzle is used to carry out the computations, which is shown in Fig. 2, where the boundary conditions are also shown. The inlet pressure is taken as 60 bar and the inlet temperature is $300 \mathrm{~K}$. The outlet pressure and

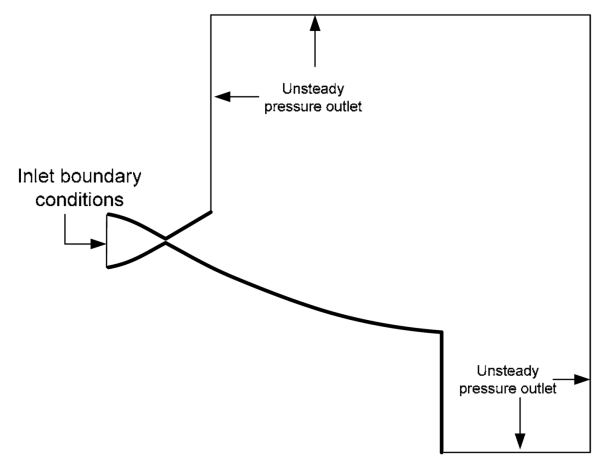

Fig. 2. Computational domain.

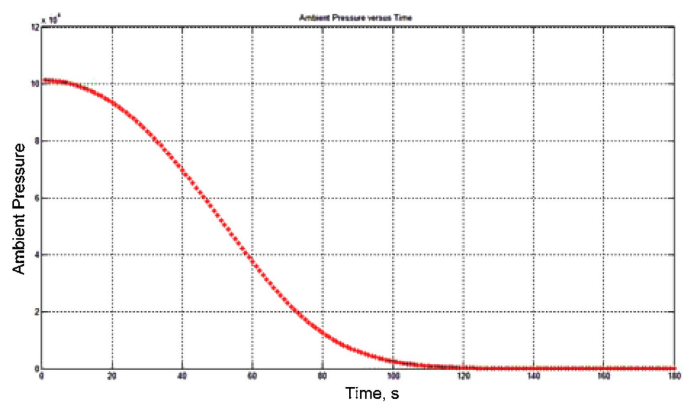

Fig. 3. Pressure variation in rocket ascending. temperature are $1.01325 \mathrm{bar}$ and $300 \mathrm{~K}$ at the ground level, and these vary with the altitude. The data is obtained from the actual flight of the PSLV rockets and the pressure variation is shown in Fig. 3. The temperature conditions for these pressures were taken from NASA atmospheric data.

The simulation is run for 179 seconds of flight. Ambient pressure goes to $0.0083 \mathrm{~Pa}$ for 179 seconds.

\subsection{Grid system}

The grid system for the computations is shown in Fig. 4. A grid independence study is also performed for the grid sensitivity to the solution. It is shown in Fig. 5. It can be seen that there is not much variation the critical property if the grid number is changed. From the study, the grid system consisting of 32312 was hence chosen for the computational analysis.

\section{Experimental Set-up}

A schlieren set-up is employed to capture the

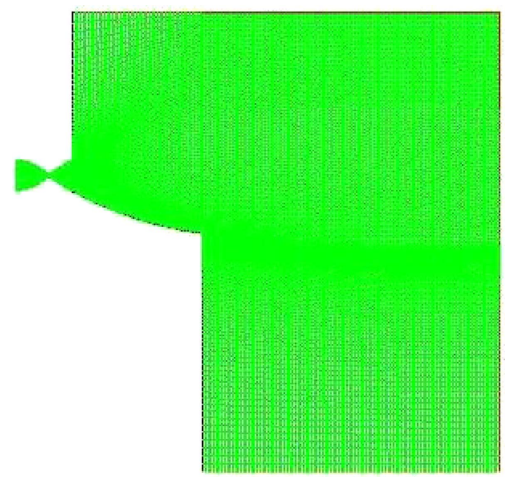

Fig. 4. Grid system.

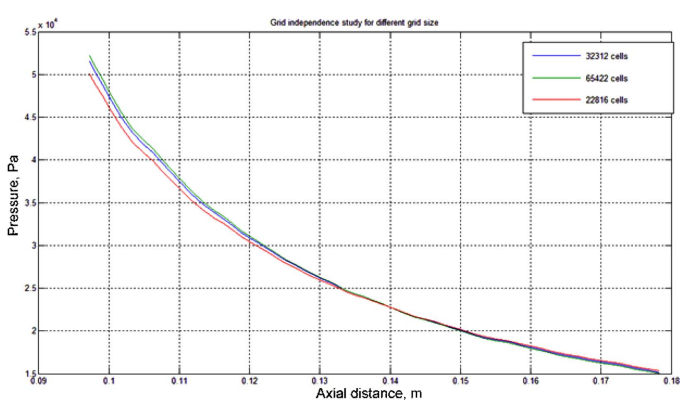

Fig. 5. Grid independence study 
density gradients in the flow field. It consists of a mono chromatic light source, two concave mirrors, a slit and a camera which is connected to a PC. A liner aerospike model, which is a straight portion of the annular model, is made from stainless steel. The experiment is done with a total pressure of 5 bar in the aerospike chamber in atmospheric conditions.

\section{Results}

The flow field of the aerospike nozzle is simulated by plotting the contours of Mach number, density and pressure in the flow field.

\section{1 validation of the results}

To validate the computations, the schlieren picture obtained through the experiment is compared with the iso-denisty contours captured in the simulations, and is shown in Fig. 6. The experimental flow field matches with the simulations reasonably well.

\subsection{Analysis of the results}

In Fig. 7, The thrust coefficient of aerospike nozzle is computed and plotted against flight time and compared with the coefficient of thrust for ideal nozzle for same total pressure.

It can be seen that the thrust coefficient of the aerospike nozzle is just lower than the ideal nozzle for most of the flight time. However, the thrust coefficient

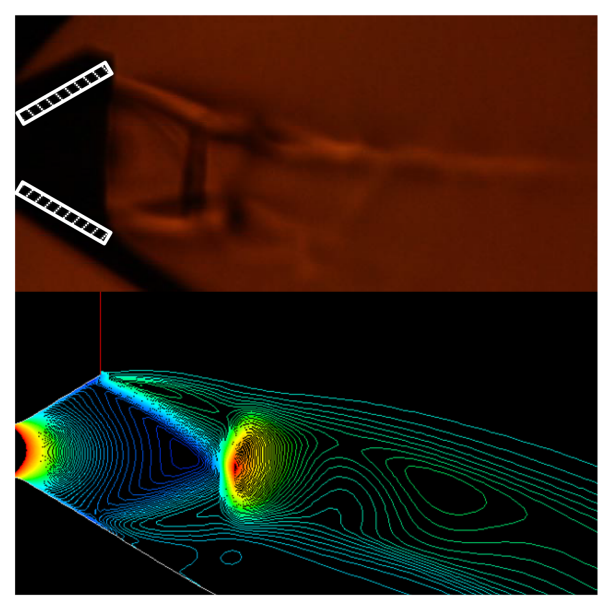

Fig. 6. Validation of the results.

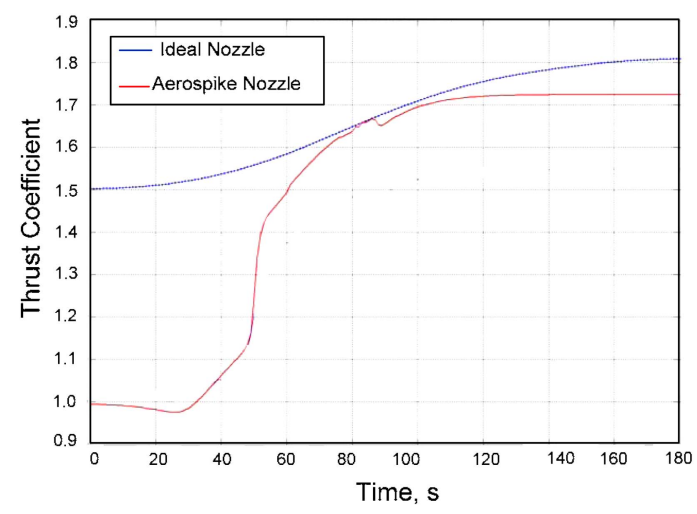

Fig. 7. Variation of coefficient of thrust.

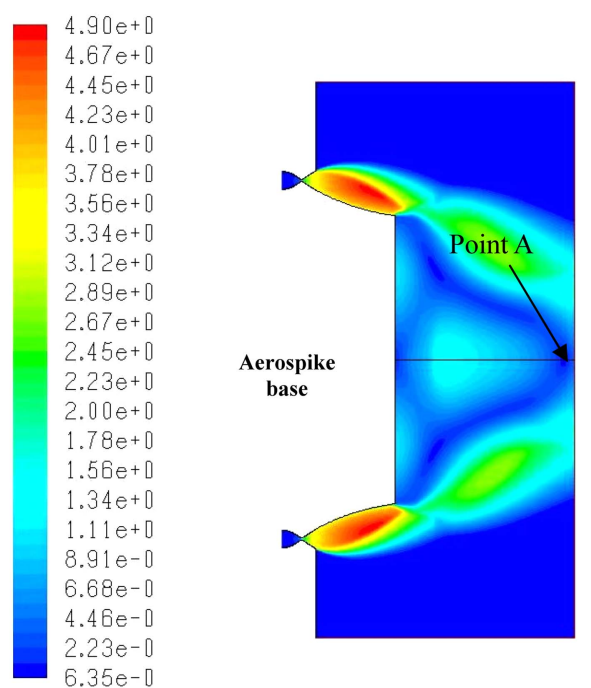

Fig. 8. Contours of Mach number $(t=0 s)$

of the aerospike nozzle is almost the same for a considerable amount of flight time.

To analyze the variation of the thrust coefficient, the Mach number and pressure contours in the full aerospike are plotted for various flight times. Fig. 8 and 9 show the Mach number and static pressure contours at the ground level $(\mathrm{t}=0 \mathrm{~s})$.

It can be seen that at the end of the wall, the flow go through a series of expansion waves and a base lip shock which ultimately increase the pressure downstream of the aerospike base. The fluid coming from both the side is meeting at point $\mathrm{A}$, losing their $\mathrm{Y}$ component of momentum and eventually the 

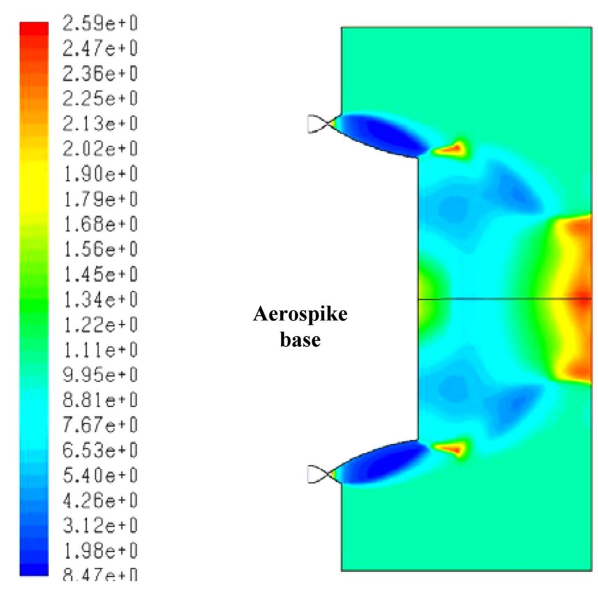

Fig. 9. Contours of pressure $(\mathrm{t}=0 \mathrm{~s})$.
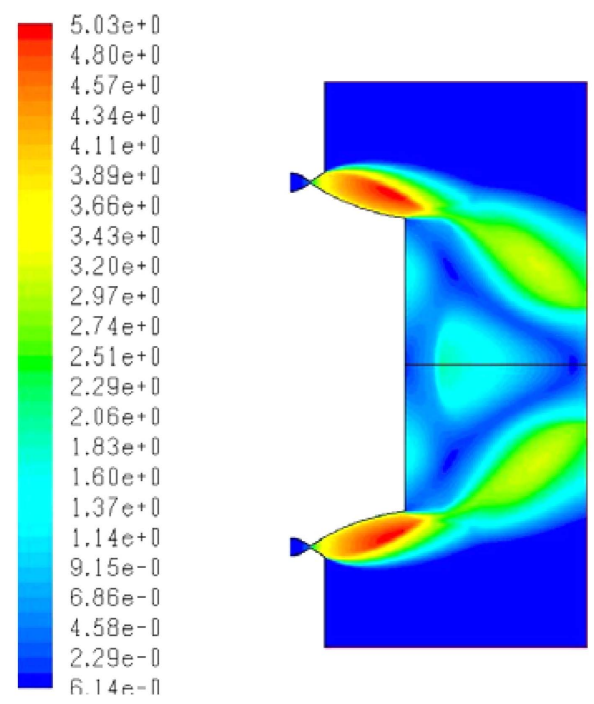

Fig. 10. Contours of Mach number $(t=30 s)$.

pressure at point $A$ increases locally and a recirculation zone is formed at the base wall. Since the static pressure at point $\mathrm{A}$ is higher than the ambient the flow must be taking place in both directions and the recirculation zone formed will be a closed one at the base of the aerospike. The stagnation point $\mathrm{A}$ is still affected by ambient pressure.

The flow field at 30s is shown in Figs. 10 and 11 where the Mach number contours and pressure contours are plotted. At $30 \mathrm{~s}$, the ambient pressure goes to $83.4 \mathrm{kPa}$ and with decrease of ambient
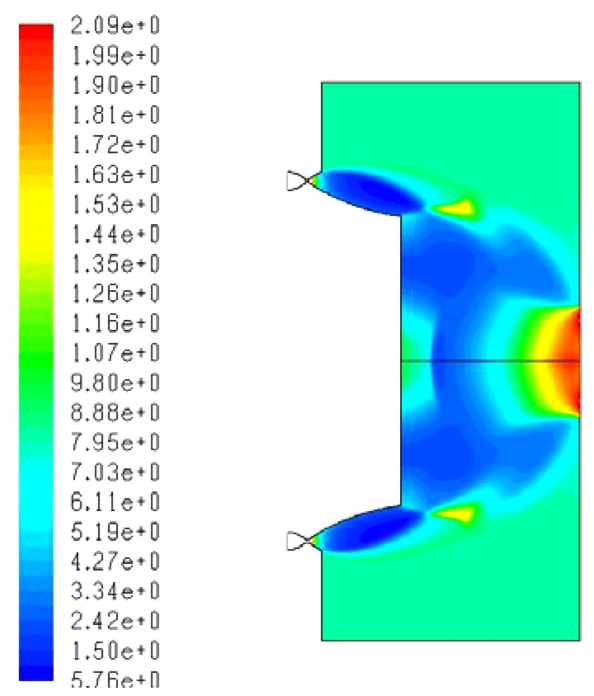

Fig. 11. Contours of Mach number $(t=30 s)$
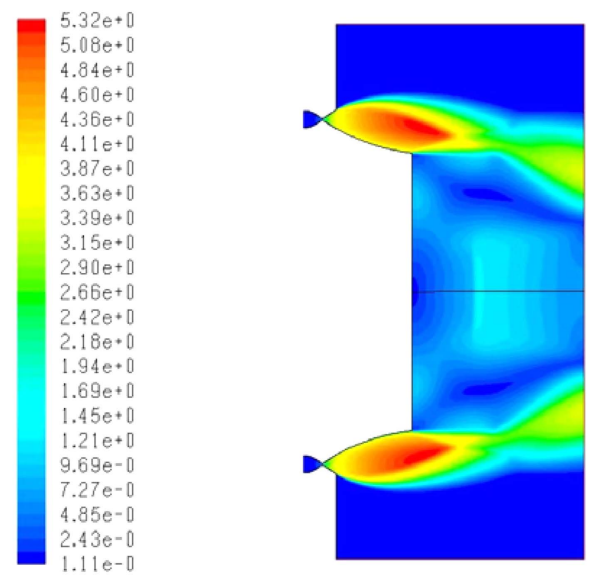

Fig. 12. Contours of Mach number $(t=50 s)$.

pressure, point A moves away from the base along Xaxis and the $\mathrm{Y}-$ momentum loss is less at point A due to reduced ambient pressure on the flow compared to the ground conditions. As a result of the loss of momentum, static pressure at point $\mathrm{A}$ increases and the recirculation zone moves away from the base wall and remains still as a closed one. The transient nature of the recirculation zone reveals that pressure on the base wall is a strong function of ambient pressure. The nozzle plumes remain to be under expanded.

Figs. 12 and 13 show the Mach number and 

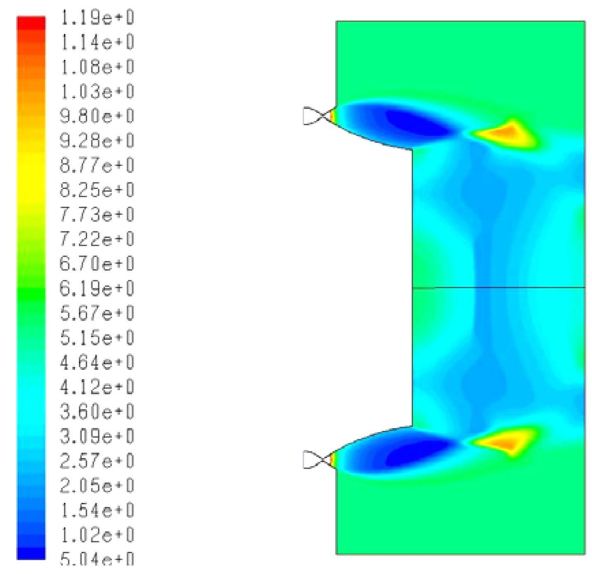

Fig. 13. Contours of static pressure $(t=50 s)$.
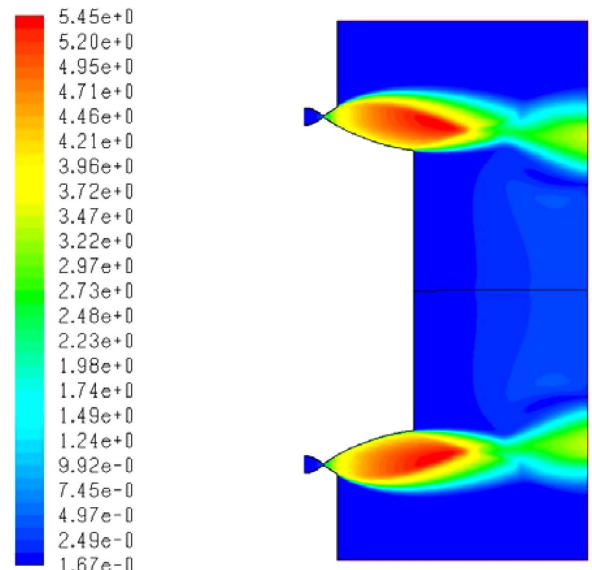

Fig. 14. Contours of Mach number $(\mathrm{t}=55 \mathrm{~s})$.

pressure contours at 50 seconds of flight. The meeting point of two jets (point A) cannot be defines now properly as it has moved far downstream along the axis. The recirculation zone becomes open now and atmospheric air starts flowing into recirculation zone.

Figs. 14 and 15 show the Mach number and pressure contours at flight time $\mathrm{t}=55 \mathrm{~s}$. At this stage, the jets will not meet and the recirculation zone disappears. The ambient air flows into the space between two jets and pressure on the wall becomes equal to the ambient pressure. At this point, the aerospike nozzle is flying at its designed pressure ratio. The ambient pressure at 55 second is $45.57 \mathrm{kPa}$.
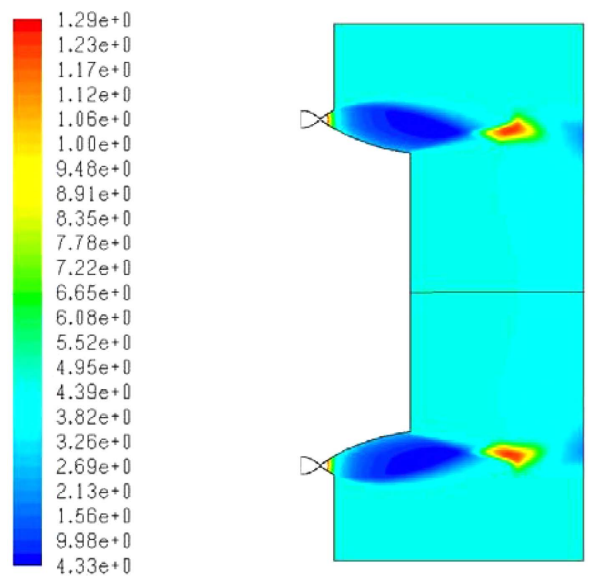

Fig. 15. Contours of static pressure $(t=55 \mathrm{~s})$.

The nozzle jets in this regime are properly expanded. The engine will operate at ideal conditions at this time and will have maximum performance. It can be seen from the Mach number and pressure contours that the jets at the lip of the lip of the nozzle do not have any shock or expansion waves.

The flow field at $100 \mathrm{~s}$ of flight time is shown in Figs.16 and 17. When the pressure goes down to a low value at this flight time, strong expansion occurs at the wall and the two jets comes nearer again as the Prandtl-

Meyer angle is large causing the jets to expand to
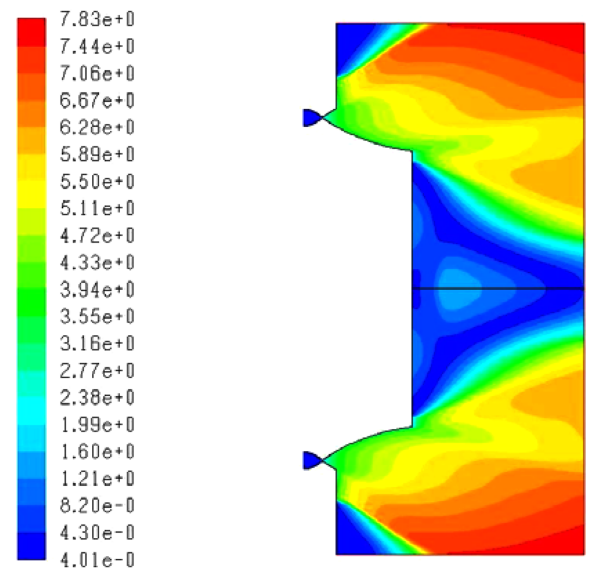

Fig. 16. Contours of Mach number $(\mathrm{t}=100 \mathrm{~s})$. 

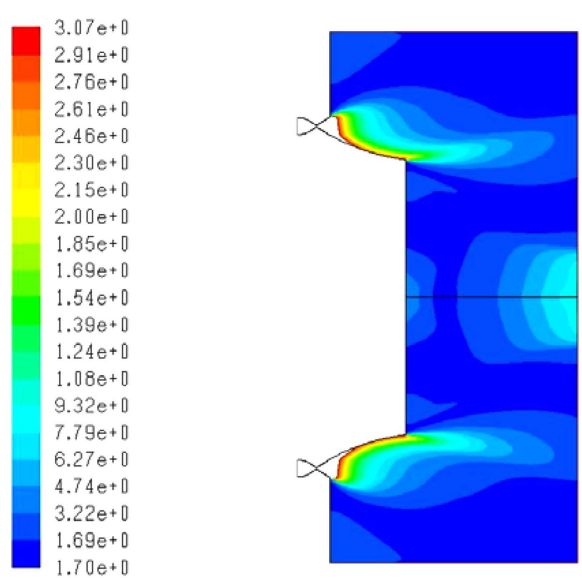

Fig. 17. Contours of static pressure $(\mathrm{t}=100 \mathrm{~s})$.

larger distances and base re-circulation zone appears once again and flow goes into closed wake condition. This time it appears in the over expanded regime of the nozzle flow. Since the closed wake flow is not affected by external flow, the pressure on the base wall due to formation of recirculation zone remains the same as earlier, where a closed wake was present. However the pressure at other side of the wall decreases due to reduced ambient pressure conditions. This contributes to an additional thrust. It should be noted here that a higher base area will result in higher thrust at this altitudes, but it will add to more drag in lower altitudes.

Based on the above analysis, the flow in the aerospike nozzle can be divides into three regimes as

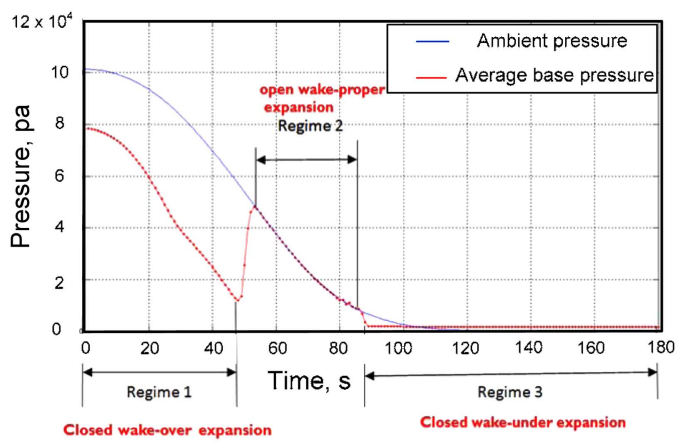

Fig. 18. Variation of base pressure and ambient pressure during flight. shown in Fig. 18, where the base pressure and the ambient pressure are plotted against the flight time. It can be seen that up to nearly $48 \mathrm{~s}$, the base pressure is less than the ambient pressure, which refers to an over expansion of the nozzle plumes. In this region, the flow from the annular aerospike exhibits a closed wake recirculation zone at its base. From $t=48 s$, to $88 \mathrm{~s}$, the base pressure becomes equal to the ambient pressure and hence the nozzle plumes are properly expanded. The wake becomes open and there is no recirculation. As shown in Fig.7, this is the flight time where the thrust coefficient of the aerospike nozzle becomes equal to that of an ideal nozzle. After $88 \mathrm{~s}$, the nozzle plumes are under expanded and strong expansion waves form at the lip of the nozzle and cause the plumes to meet again to form a closed wake region at the base of the aerospike. This region is known as the closed wake under expanded region.

\section{Conclusions}

A computational study is carried out to study the flow characteristics in an aerospike nozzle. The computational results are validated with schlieren pictures. The flow characteristics are found to be strong function of the base pressure through ambient pressure which is varying when the rocket ascends. At lower altitudes, and the over expanded plumes form a closed wake at the aerospike base. The performance of the aerospike becomes maximum when the nozzle exit pressure equals the ambient pressure at moderately high altitudes. At higher altitudes, the plumes are under expanded and the closed wake zone appears at the base and reduces the performance. Since the thrust is affected by the base pressure, a higher base area is advantageous at higher altitudes to increase the thrust of the aerospike nozzle.

\section{References}

1) Korte, J. J., 2000, "Parametric Model of an Aerospike Rocket Engine", AIAA-2000-1044.

2) Korte, J. J., Salas, A. O., Dunn, H. J., Alex, N. M., 
Follet, W. W., Oreint, G. E., and Hadid, A., H., "Multidisciplinary Approach to Aerospike nozzle design", NASA-TM- 110326.

3) George P. Shutton\& Oscar Biblarz, 2000, Rocket Propulsion Elements, Wiley India Pvt. Ltd. Seventh Edition, p. 33, 68-80.

4) Paul V. Tartabini, Roger A. Lepsch, Korte, J. J., and Kathryn E. Wurster, 2000, “A Multidisciplinary Performance Analysis of A Lifting-Body Single-Stage-
to-Orbit Vehicle", AIAA-2000-1045.

5) Verma, S. B., 2009, "Study of Restricted Shock Separation Phenomenon in a Thrust Optimized Parabolic Nozzle", J. Propulsion and Power.

6) Shannon D. Eilers, Matthew D. Wilson, and Stephen A. Whitmore, 2010, "Analytical and Experimental Evaluation of Aerodynamic Thrust Vectoring on an Aerospike Nozzle", 46th AIAA/ASME/SAE/ASEE Joint Propulsion Conf. \& Exhibit, Nashville, TN. 